\title{
Gold nanoparticles and airway hyperreactivity
}

\section{To the Editors:}

We were delighted to read the article in the European Respiratory Journal by HuSSAIN et al. [1] which demonstrated aggravation of pulmonary inflammation and airway hyperreactivity by gold (Au) nanoparticles (NPs) in a mouse model of chemical-induced asthma. Exposure of sensitised (but not unsensitised) mice to $\mathrm{Au}$ NPs was shown to result in increased airway resistance and reactivity to methacholine, associated with Au NP uptake by macrophages, increased oedema and epithelial damage. Overall, these data are consistent with the idea of a potentially toxic, exacerbating effect of nebulised Au NPs within the airway, as discussed in the editorial accompanying the article [2].

While HuSSAIN et al. [1] established the pro-inflammatory risk of $\mathrm{Au}$ NPs, the underlying mechanisms were not examined. Increased recruitment and activity of airway inflammatory cells, presumably induced by Au NP uptake, and their subsequent action is probably contributory. However, increased airway reactivity represents much more than inflammation $[3,4]$. Increased bronchoconstriction results from enhanced intracellular $\mathrm{Ca}^{2+}$ concentration $\left(\left[\mathrm{Ca}^{2+}\right]_{\mathrm{i}}\right)$ and force responses to agonist as well as airway remodelling which, in part, involves airway smooth muscle (ASM) proliferation. Accordingly, enhancement of airway reactivity by Au NPs may involve any or all of these mechanisms. We bring your attention to our recently published study [5], wherein we examined the effect of $\mathrm{Au}$ NPs on $\left[\mathrm{Ca}^{2+}\right]_{\mathrm{i}}$ and proliferation/apoptosis of human ASM cells and human bronchial epithelial cells (BECs). The aim of this in vitro study was to investigate how Au NPs interact with cells to mediate their effects. We highlighted three findings that are relevant (and supportive) to the study of HusSAIN et al. [1]: 1) positively charged Au NPs ( ${ }^{+} \mathrm{Au}$ NPs) are taken up by BECs and ASM cells within minutes, dependent on plasma membrane potential (with ${ }^{+} \mathrm{Au}$ NPs themselves inducing membrane depolarisation); 2) following uptake, only ${ }^{+} \mathrm{Au}$ NPs produce rapid elevation in $\left[\mathrm{Ca}^{2+}\right]_{\mathrm{i}}$; and 3) even $30 \mathrm{~min}$ of ${ }^{+} \mathrm{Au} \mathrm{NP}$ exposure results in substantial reduction in cell proliferation and increased apoptosis of airway cells over the next 24-48 h. We interpreted rapid depolarisation and increased $\left[\mathrm{Ca}^{2+}\right]_{i}$ induced by ${ }^{+} \mathrm{Au} \mathrm{NPs}$ in ASM as contributing to enhanced airway contractility. With ${ }^{+} \mathrm{Au}$ NP being present intracellularly, such effects could be sustained for prolonged time periods and have downstream effects of altering gene and protein regulation important to cell viability (e.g. caspases). Interestingly, Au NPs of other (or nil) charges are minimally taken up and have only small effects on $\left[\mathrm{Ca}^{2+}\right]_{i}$ or cell proliferation and survival [5]. These results raise several interesting themes relating to the results of Hussain et al. [1].

First, it may not only be the auric component of Au NPs that contributes to pulmonary inflammation and airway hyperreactivity, but Au NP charge may be important. While HuSSAIN et al. [1] did not explicitly report on Au NP charge, it would be interesting to repeat aspects of their study with nebulisation of Au NPs of different surface charges and then determine the extent of airway inflammation or reactivity.
Secondly, the effects of increased reactivity observed in mouse airways $48 \mathrm{~h}$ after Au NP nebulisation may have begun much more acutely (as evidenced by our results), and are sustained. Continued intracellular presence of Au NPs within constitutive cells of the airway (BECs and ASM) may contribute to enhanced contractility, while uptake by inflammatory cells may potentiate and perpetuate increased reactivity by modulating inflammation. It is possible that, akin to airway cells, Au NPs also increase $\left[\mathrm{Ca}^{2+}\right]_{i}$ within macrophages and other inflammatory cells, modulating pro-inflammatory versus anti-inflammatory mediator production. These issues remain to be examined.

Thirdly, the epithelial damage observed by HuSSAIN et al. [1] may very well be the result of Au NP-induced reduction of BEC viability, as observed in our study. Whether Au NPs also alter BEC function remains to be determined. In pilot studies using diaminofluorescein-based imaging in human BECs, we have not found any evidence for Au NP modulation of nitric oxide.

A major contextual difference between our study and that of HuSSAIN et al. [1] (beyond species differences and our study being in vitro only) is that in the latter study, Au NP exposure occurred in an environment of pre-existing inflammation. It would be interesting to determine whether early pro-inflammatory cytokines, such as tumour necrosis factor- $\alpha$ or interleukin-1 $\beta$, alter the extent of Au NP (especially ${ }^{+} \mathrm{Au}$ NP) uptake and its downstream consequences.

Finally, from the perspective of Au NP toxicity, based on our study, we would conclude that "charge matters". Accordingly, in designing $\mathrm{Au} \mathrm{NP}$-containing preparations, we propose that attention be paid to Au NP charge. Here, the lack of toxic effect of non-positive Au NPs suggests the need to explore favourable modification of Au NP charge and the maintenance of such charge within biologically compatible formulations of drugs and other substances for human use.

\section{R. Arvizo*, M.A. Thompson", C.M. Pabelick ${ }^{\#,}$, , V. Rotello ${ }^{+}$, P. Mukherjee*, and Y.S. Prakash ${ }^{\#, \oplus}$ \\ Depts of *Biochemistry and Molecular Biology, "Anesthesiology and "Physiology and Biomedical Engineering, College of Medi- cine, Mayo Clinic, Rochester, MN, and 'Dept of Chemistry, University of Massachusetts, Amherst, MA, USA.}

Correspondence: Y.S. Prakash, Dept of Anesthesiology, 4-184 W Jos SMH, 200 First St SW, Mayo Clinic, Rochester, MN 55905, USA. E-mail: prakash.ys@mayo.edu

Statement of Interest: None declared.

\section{REFERENCES}

1 Hussain S, Vanoirbeek JAJ, Luyts K, et al. Lung exposure to nanoparticles modulates an asthmatic response in a mouse model. Eur Respir J 2011; 37: 299-309.

2 Lanone S, Boczkowski J. Titanium and gold nanoparticles in asthma: the bad and the ugly. Eur Respir J 2011; 37: 225-227. 
3 Mahn K, Ojo OO, Chadwick G, et al. $\mathrm{Ca}^{2+}$ homeostasis and structural and functional remodelling of airway smooth muscle in asthma. Thorax 2010; 65: 547-552.

4 Meurs H, Gosens R, Zaagsma J. Airway hyperresponsiveness in asthma: lessons from in vitro model systems and animal models. Eur Respir J 2008; 32: 487-502.
5 Arvizo RR, Miranda OR, Thompson MA, et al. Effect of nanoparticle surface charge at the plasma membrane and beyond. Nano Lett 2010; 10: 2543-2548.

\section{Hypersensitivity pneumonitis and metalworking fluids contaminated by mycobacteria}

\section{To the Editors:}

We read with interest the article published by TILLIE-LEBLOND et al. [1] relating to hypersensitivity pneumonitis (HP) in French automobile workers exposed to metalworking fluids (MWFs). Our group was involved in the UK outbreak investigation referenced in their article [2, 3], and have a clinical and research interest in this area.

Whilst TILLIE-Leblond et al. [1] are correct in stating that the majority of MWF-HP outbreaks have occurred in the USA, the UK Powertrain and French outbreaks are not the only ones to have occurred in Europe. We have provided scientific support to three other similar outbreaks in the UK, all with confirmed cases of MWF-HP [4, 5]. In addition, we have diagnosed single cases of MWF-HP or asthma in workers from at least five other workplaces close to our occupational lung disease clinic. We are also aware of a published respiratory outbreak from Croatia [6], and have recently had separate personal communications with groups in Germany and Sweden relating to investigations of possible MWF outbreaks. Supportive evidence for a more widespread European problem come from cross-sectional studies that have demonstrated an excess of respiratory symptoms and asthma in machine shop workers in southern Finland [7], and an excess of wheeze, chronic bronchitis, chronic rhinitis and eye irritation in Swedish metalworkers [8].

It is clear, therefore, that this remains an important area of research relevant to European MWF-exposed workers, as the exact aetiology of MWF-HP has remained elusive [9] since BERNSTEIN et al. [10] described the first cases in 1995. Although Mycobacterium immunogenum have been implicated as the cause in the French outbreak and a number of outbreaks in the USA, there is strong evidence against this being the cause of the UK outbreaks [3,5]. In the UK Powertrain investigation, it was not possible to culture any opportunuistic mycobacteria or find any evidence of mycobacterial DNA by PCR in 125 MWF samples. Analysis of MWF samples from two other workplaces associated with UK MWF-HP outbreaks also found no detectable mycobacterial DNA. In addition to this, no demonstrable precipitin responses to extracts of $M$. immunogenum, Mycobacterium chelonae or Mycobacterium fortuitum were seen in 129 Powertrain UK workers, a group that included 17 cases of MWF-HP, 70 cases of occupational asthma, and 42 asymptomatic exposed controls. Further evidence against a mycobacterial cause came from specific challenges performed in two workers, where positive responses were seen after controlled exposure to used MWFs that did not contain mycobacteria [3].

Although referenced by TILLIE-LEBLOND et al. [1], the detailed immunological investigation performed on workers from a MWF-HP outbreak in the USA, where mycobacterial contamination was identified [11], is not discussed in any detail. In this key study [11], in vitro secretion of interleukin-8, tumour necrosis factor- $\alpha$ and interferon- $\gamma$ were measured in whole blood and from peripheral blood mononuclear cells in response to incubation with $M$. immunogenum antigen. These measures of immunity to $M$. immunogenum were able to distinguish between MWF-exposed and -unexposed workers, but not between workers with and without MWF-HP. This study serves to highlight the difficulties of interpreting immunological findings in $\mathrm{HP}$, as many workers with demonstrable immune responses are asymptomatic, and never go on to develop disease [12].

TilLiE-Leblond et al. [1] state in their article that the presence of five arcs to $M$. immunogenum by electrosyneresis differentiates MWF-HP from healthy exposed workers. Whilst this may have been true in most cases, it was not true for all, and may therefore be of limited clinical value in isolation. It should be noted that the two groups in this study had also been differentiated in other ways, based on the presence of symptoms, lung crackles and normal gas transfer prior to any immunological comparison. Whilst we agree with the authors that this test threshold needs further validation, there seems little point in aiming to differentiate those with MWFHP from healthy workers without symptoms, as this can be done more easily by questionnaire. Whether the five-arc threshold has any diagnostic value is dependant on whether it can assist in differentiating workers experiencing workrelated symptoms due to allergic occupational lung disease (HP and asthma) from those with similar symptoms due to chronic bronchitis, exacerbations of existing respiratory conditions, nonspecific irritant responses or humidifier fever. Alternatively, the utility of the test in preventing disease, as part of a prospective study of health surveillance linked to exposure modification in MWF apprentices, would also be of great interest. It is these areas that we believe merit further study by the authors to continue to improve the knowledge base in the complex area of MWF-HP outbreaks. 